\title{
Leisure-Time Physical Activity and Cardiovascular Mortality in an Elderly Population in Northern Manhattan: A Prospective Cohort Study
}

\author{
Ying Kuen Cheung, $P h D^{7}$, Yeseon P. Moon, $\mathrm{MS}^{2}$, Erin R. Kulick, MPH ${ }^{2}$, Ralph L. Sacco, MD, $M S^{3}$, \\ Mitchell S. V. Elkind, MD, $M S^{2,4}$, and Joshua Z. Willey, MD, $M S^{2}$ \\ 'Department of Biostatistics, Columbia University, New York, NY, USA; ${ }^{2}$ Department of Neurology, Columbia University, New York, NY, USA; \\ ${ }^{3}$ Department of Neurology, University of Miami, New York, NY, USA; ${ }^{4}$ Department of Epidemiology, Columbia University, New York, NY, USA.
}

\begin{abstract}
BACKGROUND: Previous studies of exercise have focused on measuring physical activity in totality using summary statistics such as metabolic equivalent score for total intensity or total energy count.

OBJECTIVE: We aimed to examine the multidimensionality of leisure-time physical activity (LTPA) and to identify the specific LTPA components that were associated with cardiovascular mortality in the elderly.

DESIGN AND PARTICIPANTS: The Northern Manhattan Study (NOMAS) is a multiethnic prospective cohort of elderly stroke-free individuals consisting of a total of 3298 participants recruited between 1993 and 2001, with a median follow-up of 17 years.
\end{abstract}

MAIN MEASURES: Physical activity questionnaire data were available in 3293 NOMAS participants, who were categorized into subgroups with similar exercise patterns by model-based cluster analysis. Three subgroupdefining LTPA features were identified and were considered as primary exposures in Cox proportional hazard models: frequency of activity, number of activity types (variety), and energy-to-duration ratio (EDR). We considered cardiovascular mortality and non-cardiovascular mortality as outcomes in Cox cause-specific proportional hazard models, and all-cause mortality as outcome in Cox models.

KEY RESULTS: A high activity frequency was associated with reduced cardiovascular mortality (hazard ratio, $\mathrm{HR}=0.93, P=0.03$ ), but demonstrated no effect on non-cardiovascular death. A high EDR was associated with increased risk of cardiovascular death $(\mathrm{HR}=$ 1.30, $P=0.01)$. A high number of activity types was beneficial in reducing all-cause mortality $(\mathrm{HR}=0.87$, $P=0.01)$.

CONCLUSIONS: Exercise frequency was protective against cardiovascular mortality, and a high variety of activity was protective against all-cause mortality. The performance of frequent and varied non-intense exercise in an elderly population such as ours is achievable and can reduce the risk of death.

Received May 13, 2016

Revised July 19, 2016

Accepted September 19, 2016

Published online October 17, 2016
KEY WORDS: exercise; mortality; cardiovascular.

J Gen Intern Med 32(2):168-74

DOI: $10.1007 / \mathrm{s} 11606-016-3884-y$

(c) Society of General Internal Medicine 2016

\section{INTRODUCTION}

Numerous epidemiological studies and meta-analyses have demonstrated the protective effects of exercise and leisuretime physical activity (LTPA) against cardiovascular disease and mortality across all age and demographic groups. ${ }^{1-6}$ However, even though there are many facets of physical activity, the existing literature focuses mostly on total energy count or total intensity in terms of metabolic equivalent score as the basis for recommendations. This approach offers little insight into how particular aspects of physical activity affect cardiovascular risks, and potentially limits the clinical effectiveness of recommendations, especially because there is evidence that the benefits may be achieved at lower levels of activity. ${ }^{7,8}$ In addition, when providing lifestyle change recommendations to patients, giving more specifics as to frequency, duration, and intensity may be more successful at altering behavior compared to generic advice. For example, while the American Heart Association guidelines recommend 150 min of moderate intensity or $75 \mathrm{~min}$ of heavy intensity per week for primary prevention of cardiovascular disease, ${ }^{9}$ further specifics as to how to achieve these goals are not well known.

The Northern Manhattan Study (NOMAS) is a multiethnic prospective cohort of elderly stroke-free individuals, and has served as a resource for studying determinants of cardiovascular disease risk in this unique demographic. In order to provide a better understanding of the LTPA patterns in NOMAS, in a previous work, we used model-based cluster analysis to divide the cohort into subgroups of subjects with similar exercise patterns. ${ }^{10}$ The use of clustering algorithms enabled us to use multidimensional LTPA measures as inputs and to produce homogeneous subgroups in a data-driven manner. ${ }^{11}$ In the current study, we explored the LTPA features that characterize these previously derived subgroups. We then examined the association between these subgroup-defining features and mortality (cardiovascular, non-cardiovascular, and all-cause) in order to better describe the impacts of LTPA 
on these outcomes. Our goal was to generate information that allows providers to give more specific counseling to patients beyond a single index of total weekly activity.

\section{METHODS}

\section{Study Population}

The NOMAS is a population-based cohort study designed to evaluate the effects of medical, socioeconomic, and other risk factors on the incidence of cardiovascular disease in a strokefree multiethnic cohort. Methods of participant recruitment, evaluation, and follow-up have been previously reported. ${ }^{12} \mathrm{In}$ person evaluations were performed at Columbia University Medical Center or at home for those who could not come in person $(6 \%)$. The study was approved by the institutional review boards at Columbia University Medical Center and the University of Miami. All participants gave informed consent to participate in the study.

The NOMAS cohort consists of a total of 3298 participants recruited between 1993 and 2001, with mean age of 69 years ( \pm 10 years) at baseline.

\section{Physical Activity Features}

Baseline physical activity was measured by an in-person questionnaire adapted from the National Health Interview Survey of the National Center for Health Statistics. ${ }^{13}$ The questionnaire consisted of 14 pre-specified activities, including walking, jogging or running, hiking, gardening or yard work, aerobics or aerobic dancing, other dancing, calisthenics or general exercise, golf, tennis, bowling, bicycle riding, swimming or water exercise, horseback riding, handball, racquetball, and squash. In addition, two open fields were allowed for activities that were not listed. We kept the open-ended activities separate to account for the diversity of the activities performed. For each activity, participants were asked for the following self-report variables: participation in the activity type, the frequency of each activity during the prior 2-week period, and the duration of the activity at each session. The questionnaire was previously found to be reliable for individuals reporting moderate physical activity and was validated in this population. ${ }^{13}$

Questionnaires were correlated with compendia of physical activity to allow calculation of metabolic equivalents for the intensity of activity and energy expenditure in kilocalories. ${ }^{14}$ The metabolic equivalent score for an activity by a participant was calculated as the product of metabolic equivalents and activity duration. For each participant, we then derived the total metabolic equivalent score (METS) by adding all activity-specific metabolic equivalence scores, the total energy expenditure for all activities in the prior 2 weeks, the total frequency of physical activity sessions in the same period, the average duration per session, and the number of activity types. Based on these LTPA measures, the participants were grouped into five subgroups using multivariate finite mixture modeling, in addition to a no-activity group, resulting in a total of six LTPA subgroups. The details of the methodology have been described elsewhere. ${ }^{10}$

While our previous work produced subgroups of subjects characterized by similar exercise patterns without considering health outcomes, the current study identified three subgroupdefining LTPA features: weekly activity frequency, number of activity types (variety), and energy-to-duration ratio (EDR). The EDR was defined as the ratio between total weekly energy expenditure and the average duration per session, and can be equivalently calculated as $E D R=$ Average energy expended per hour during exercise $\times$ frequency of exercise. For example, if an individual exercises four times a week for a total of $800 \mathrm{kcal}$ over a 2-h period during the week, then the average energy is calculated as $800 \mathrm{kcal} \div 2 \mathrm{~h}=400 \mathrm{kcal} / \mathrm{h}$, and the EDR will equal $400 \mathrm{kcal} / \mathrm{h} \times 4=1600 \mathrm{kcal} / \mathrm{h}$. The purpose of this study was to assess the association between these LTPA features and mortality.

\section{Baseline Risk Factors}

Data regarding baseline status and risk factors were collected through participant interviews. Race/ethnicity was determined by self-identification based on a questionnaire adapted from the 2000 US census, and conformed to the standard definitions outlined by Directive $15 .{ }^{15}$ Education was categorized as completing high school versus not completing high school. Standardized questions were asked regarding hypertension, diabetes, cigarette smoking, and cardiac condition. ${ }^{16}$ Smoking status was classified as current smoker (within the past year), former smoker, and never smoker. Standard techniques were used to measure height, weight, and blood pressure. Diabetes mellitus was defined as fasting blood glucose $\geq 126 \mathrm{mg} / \mathrm{dL}$ or higher, the patient's self-report of diabetes mellitus, or insulin or hypoglycemic agent use. Hypertension was defined as systolic blood pressure $\geq 140 \mathrm{mmHg}$ or diastolic blood pressure $\geq 90 \mathrm{mmHg}$ based on the average of two blood pressure measurements, physician diagnosis, or patient self-report of a history of hypertension or antihypertensive drug use. Moderate alcohol consumption was defined as $>1$ alcoholic drink per month and $\leq 2$ drinks per day. Participant insurance type, used as another proxy for socioeconomic status, was classified as no insurance or Medicaid versus Medicare or private insurance.

\section{Cardiovascular Outcomes and Mortality}

Participants were followed up annually via phone screening to detect any new cardiac or neurological symptoms, hospitalizations, medical conditions, or death. Myocardial infarction (MI) was defined by criteria adapted from the Cardiac Arrhythmia Suppression Trial and the Lipid Research Clinics Coronary Primary Prevention Trial. ${ }^{17}$ Sudden cardiac death was defined as death due to cardiac arrest, or sudden unexplained death discovered within $24 \mathrm{~h}$ of the event. ${ }^{18}$ 
Neurological events that were possible strokes were adjudicated independently by two neurologists after review of all data. Cardiovascular death was defined as all deaths arising from stroke, MI, congestive heart failure, pulmonary embolism, arrhythmia/sudden death, aortic aneurysm, aortic or mitral stenosis, or left ventricular hypertrophy, which were considered cardiovascular death endpoints. Deaths ruled not of cardiovascular origin were deemed non-cardiovascular, and deaths without sufficient records were deemed of unknown etiology. ${ }^{19}$ Cause of death was ascertained through phone discussion with the participant's family, review of medical records, and when available, a copy of the death certificate. Complete loss to follow-up occurred in less than $1 \%{ }^{20}$

\section{Statistical Analysis}

The associations between each of the three LTPA features and other baseline risk factors were described by summary statistics (Pearson's correlation coefficient, means, and standard deviations); $P$ values were reported to assess significance. Causespecific hazards (cardiovascular and non-cardiovascular) were assessed using Cox models that include the three LTPA features, adjusting for other baseline variables and risk factors. The covariates in the models were age, BMI, sex, race/ethnicity, education, smoking status, alcohol consumption, insurance type, diabetes, hypertension, and cardiac disease. All-cause mortality risk was also assessed using Cox models. Smoothing splines were used to explore possible non-linear LTPA effects. These analyses excluded participants who did not have data on physical activity data $(n=5)$ or who had pre-existing MI at baseline $(n=244)$. An additional 39 participants had missing baseline status of education $(n=2)$, diabetes $(n=6)$, BMI $(n=$ $11)$, or insurance type $(n=20)$. Thus, the Cox regression analyses included 3010 participants in the cohort. LTPA features of these 39 participants with missing covariates were compared to those of the 3010 with complete records. All analyses were performed using $\mathrm{R}$ version 3.1.2.

\section{RESULTS}

Figure 1 shows two scatterplots of four LTPA measures colorcoded by subgroup number. The subgroups are visually distinguished by activity frequency and also gradually associated with the number of activity types (Fig. 1, right). In contrast, the individual subgroups do not seem to be characterized by a distinctive pattern in terms of weekly energy expenditure or average session duration per se, but are clearly defined by the ratio between these two measures, indicated by the slope (Fig. 1, left). This energy-to-duration ratio (EDR) and the frequency and number of types of activity were used as the LTPA features in the association analyses.

In our cohort, the mean activity frequency was 3.6 times per week (standard deviation, $\mathrm{SD}=4.2$ ), mean EDR was $1133 \mathrm{kcal}$ per hour $(\mathrm{SD}=1432 \mathrm{kcal} / \mathrm{h})$, and mean number of activity types was $0.8(\mathrm{SD}=0.9)$. Table 1 describes how these LTPA
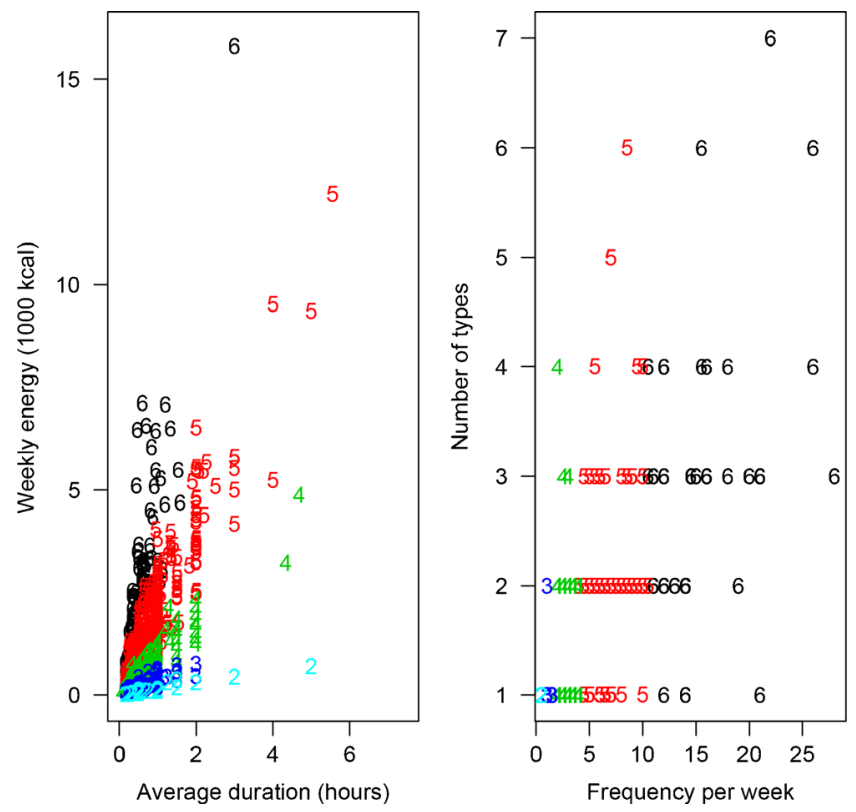

Fig. 1 Characteristics of leisure-time physical activity in NOMAS participants. The numbers indicate the subgroups to which participants belong. Participants in subgroup 1 reported no physical activity, i.e., having all physical activity measures equal to 0 . (These plots use data from 1546 random samples of the 3021 subjects, because the plots using all subjects were too large to transmit).

features correlate with other baseline characteristics. The mean levels of these features were concordant with each other for each factor. For example, men had higher frequency, EDR, and variety compared to women. Other factors that were positively associated with all three LTPA features were nonHispanic white race/ethnicity, at least a high school education level, former smoker status, moderate alcohol consumption, having Medicare or private insurance, absence of diabetes, and absence of hypertension. In addition, a high BMI was associated with low frequency and number of activity types, and high EDR. There was no difference in the means of these LTPA features between the 3010 participants included in this analysis and participants with missing baseline values.

Among the 3010 participants, with a median follow-up of 17 years, there were 625 confirmed cardiovascular deaths. Table 2 shows the results of Cox models that assess the joint effects of the three LTPA features, after adjusting for other baseline variables. A high frequency of activity reduced the risk of cardiovascular death ( $\mathrm{HR}=0.93$ per session, $P=0.03$ ), whereas high EDR was associated with an increased risk $(\mathrm{HR}=1.30$ per $1000 \mathrm{kcal} / \mathrm{h}$ EDR, $P=0.01)$. The effect a of high number of activity types (variety) did not reach statistical significance, but otherwise appeared to suggest a protective trend against cardiovascular mortality $(\mathrm{HR}=0.85$ per activity type, $P=0.06$ ). In addition to the 625 confirmed cardiovascular deaths, there were 128 deaths of unknown etiology. As a sensitivity analysis, Cox regression was performed assuming these 128 events were cardiovascular mortality, and gave essentially identical results. Figure 2 shows no evidence of non-linear LTPA effects on cardiovascular mortality $(P=0.74$, 
Table 1 Association Between LTPA Features and Baseline Characteristics in NOMAS Participants

\begin{tabular}{|c|c|c|c|}
\hline \multirow[t]{2}{*}{ Characteristic (n) } & \multicolumn{3}{|c|}{ LTPA feature } \\
\hline & $\begin{array}{l}\text { Activity } \\
\text { frequency }\end{array}$ & EDR & $\begin{array}{l}\text { Number } \\
\text { of activity } \\
\text { types }\end{array}$ \\
\hline \multicolumn{4}{|c|}{ Correlation between LTPA and continuous characteristics } \\
\hline Age & 0.03 & -0.02 & -0.02 \\
\hline BMI & $-0.10 t$ & $0.04 *$ & $-0.07 \$$ \\
\hline \multicolumn{4}{|c|}{ Mean (SD) of LTPA by categorical characteristics } \\
\hline \multicolumn{4}{|l|}{ Sex } \\
\hline Female (1909) & $3.3(4.0) \div$ & $990(1267) \ddagger$ & $0.80(0.85)$ \\
\hline Male (1101) & $4.0(4.5) \div$ & $1372(1651) \ddagger$ & $0.91(0.91)$ \\
\hline \multicolumn{4}{|l|}{ Race/ethnicity } \\
\hline White (607) & $4.7(4.5) \ddagger$ & $1424(1422) \ddagger$ & $1.07(0.99) \ddagger$ \\
\hline Black (732) & $4.1(4.1)+$ & $1327(1423) \ddagger$ & $0.91(0.83)$ \\
\hline Hispanic (1602) & $2.9(3.9) \div$ & $912(1378)+$ & $0.70(0.80)$ \\
\hline \multicolumn{4}{|l|}{ Education } \\
\hline No high school (1631) & $2.9(3.8) \div$ & $908(1260)+$ & $0.69(0.75)$ \\
\hline At least high school (1379) & $4.3(4.5) \div$ & $1391(1571) \dagger$ & $1.01(0.98)$ \\
\hline \multicolumn{4}{|l|}{ Smoking status } \\
\hline Never smoker (1422) & $3.4(4.0)$ & $1047(1297) *$ & $0.83(0.85)$ \\
\hline Former smoker (1073) & $3.9(4.5)$ & $1279(1608)^{*}$ & $0.89(0.94)$ \\
\hline Current smoker (515) & $3.4(4.0)$ & $1049(1368) *$ & $0.75(0.81)$ \\
\hline \multicolumn{4}{|l|}{ Moderate alcohol consumption } \\
\hline No $(1993)$ & $3.2(3.9) \div$ & $996(1254) \ddagger$ & $0.74(0.77) \ddagger$ \\
\hline Yes (1017) & $4.3(4.7)+$ & $1391(1697) \ddagger$ & $1.03(1.02)$ \\
\hline \multicolumn{4}{|l|}{ Insurance type } \\
\hline Medicare or private (1688) & $4.0(4.3) \$$ & $1275(1455) \ddagger$ & $0.93(0.90) \ddagger$ \\
\hline Medicaid or none (1322) & $3.0(4.0) \div$ & $943(1378)+$ & $0.72(0.83) \ddagger$ \\
\hline \multicolumn{4}{|l|}{ Diabetes } \\
\hline No (2379) & $3.7(4.3) \dagger$ & $1159(1447) \dagger$ & $0.87(0.90) \dagger$ \\
\hline Yes (631) & $3.1(3.9) \dagger$ & $1016(1363) \dagger$ & $0.72(0.76) \dagger$ \\
\hline \multicolumn{4}{|l|}{ Hypertension } \\
\hline No $(827)$ & $4.0(4.5) \dagger$ & $1216(1475)^{*}$ & $0.93(0.95) \dagger$ \\
\hline Yes (2183) & $3.4(4.1) \dagger$ & $1097(1413)^{*}$ & $0.81(0.84) \dagger$ \\
\hline \multicolumn{4}{|l|}{ Cardiac disease } \\
\hline No $(2556)$ & $3.6(4.2)$ & $1135(1451)$ & $0.84(0.89)$ \\
\hline Yes (454) & $3.5(4.0)$ & $1099(1317)$ & $0.81(0.77)$ \\
\hline
\end{tabular}

For continuous characteristics, $P$ values test whether the correlation with LTPA is zero, based on Fisher's $Z$ transform. For categorical characteristics, $P$ values test whether mean LTPA is different among groups based on the Kruskal-Wallis test. EDR energy-to-duration ratio, LTPA leisure-time physical activity, NOMAS Northern Manhattan Study

$* P<0.05$

$+P<0.01$

$\neq P<0.001$

$0.12,0.41$, respectively, for frequency, EDR, and number of types).

Table 2 also shows the results of Cox regression models estimating the risk of non-cardiovascular (907 events) and all-cause mortality (1532 events). Frequency and EDR were not associated with risk of non-cardiovascular death $(P=0.64,0.64)$, but were marginally associated with allcause mortality $(P=0.08,0.05)$. The effect of LTPA variety did not reach significance for non-cardiovascular death (HR $=0.88, P=0.06)$, but was protective against all-cause mortality $(\mathrm{HR}=0.87, P=0.01)$. In a post hoc analysis, average energy expended was added to the model on cardiovascular mortality. After accounting for EDR (which is an interaction between frequency and average energy expended), the average energy expended was not associated with cardiovascular mortality $(P=0.9)$, while EDR remained significant $(P=0.01)$. The effect sizes of LTPA variety on cardiovascular and non-cardiovascular deaths were similar. This, together with the fact that high variety was protective against all-cause mortality, with similar effect size, suggests that the insignificant results for cause-specific mortality might be due to inadequate power.

\section{DISCUSSION}

The cardioprotective effects of LTPA have been established across different demographic groups, ${ }^{21}$ and yet there is relatively little clarity on which aspect of LTPA is beneficial specifically for cardiovascular mortality, and which has an overall benefit non-specific to cardiovascular health. Physical activity is a multidimensional construct that includes intensity, frequency, duration, and variety, but prior studies have rarely considered all these aspects in combination. The present analysis sheds light on the specific effects of frequency and intensity of physical activity on cardiovascular mortality in a racially/ethnically diverse elderly population, as evidenced by the lack of association with non-cardiovascular death. Cause-specific hazard models were used to address the 
Table 2 Mortality Risk (Cardiovascular, Non-Cardiovascular, All-Cause) of LTPA Features and Other Baseline Demographic and Cardiovascular Risk Factors

\begin{tabular}{|c|c|c|c|c|c|c|}
\hline & \multicolumn{2}{|c|}{ Cardiovascular $(n=625)$} & \multicolumn{2}{|c|}{$\begin{array}{l}\text { Non-cardiovascular } \\
(n=907)\end{array}$} & \multicolumn{2}{|c|}{ All-cause $(n=1532)$} \\
\hline & HR & CI & HR & CI & HR & CI \\
\hline Frequency & 0.93 & $0.86-0.99$ & 0.99 & $0.93-1.05$ & 0.96 & $0.92-1.00$ \\
\hline Energy-duration ratio (EDR) & 1.30 & $1.06-1.59$ & 1.05 & $0.87-1.26$ & 1.15 & $1.00-1.32$ \\
\hline Number of types (variety) & 0.85 & $0.72-1.01$ & 0.88 & $0.77-1.00$ & 0.87 & $0.78-0.96$ \\
\hline Age & 1.11 & $1.10-1.12$ & 1.09 & $1.08-1.10$ & 1.10 & $1.09-1.10$ \\
\hline Male & 1.34 & $1.12-1.62$ & 1.60 & $1.38-1.85$ & 1.49 & $1.33-1.67$ \\
\hline \multicolumn{7}{|l|}{ Race/ethnicity } \\
\hline Black & 0.88 & $0.71-1.10$ & 1.13 & $0.94-1.35$ & 1.02 & $0.89-1.17$ \\
\hline Hispanic & 0.52 & $0.40-0.67$ & 0.68 & $0.55-0.85$ & 0.61 & $0.51-0.72$ \\
\hline \multicolumn{7}{|l|}{ Education } \\
\hline At least high school & 0.79 & $0.65-0.96$ & 0.88 & $0.75-1.03$ & 0.84 & $0.74-0.95$ \\
\hline \multicolumn{7}{|l|}{ Smoking status } \\
\hline Former smoker & 1.06 & $0.89-1.27$ & 1.30 & $1.11-1.51$ & 1.19 & $1.06-1.34$ \\
\hline Current smoker & 1.44 & $1.13-1.84$ & 1.99 & $1.66-2.40$ & 1.76 & $1.52-2.03$ \\
\hline Moderate alcohol consumption & 0.88 & $0.74-1.05$ & 0.86 & $0.74-1.00$ & 0.87 & $0.78-0.98$ \\
\hline Medicaid or no insurance & 1.13 & $0.93-1.39$ & 1.12 & $0.96-1.32$ & 1.13 & $1.00-1.28$ \\
\hline Diabetes & 1.56 & $1.29-1.88$ & 1.43 & $1.22-1.67$ & 1.48 & $1.31-1.67$ \\
\hline Hypertension & 1.24 & $1.01-1.52$ & 0.98 & $0.84-1.14$ & 1.07 & $0.95-1.21$ \\
\hline Cardiac disease & 1.80 & $1.49-2.17$ & 1.15 & $0.96-1.38$ & 1.40 & $1.23-1.60$ \\
\hline BMI & 0.99 & $0.98-1.01$ & 0.99 & $0.97-1.00$ & 0.99 & $0.98-1.00$ \\
\hline
\end{tabular}

$P$ values associated with the LTPA features are not shown in the table but are reported in the text LTPA leisure-time physical activity, HR hazard ratio, CI confidence interval, BMI body mss index
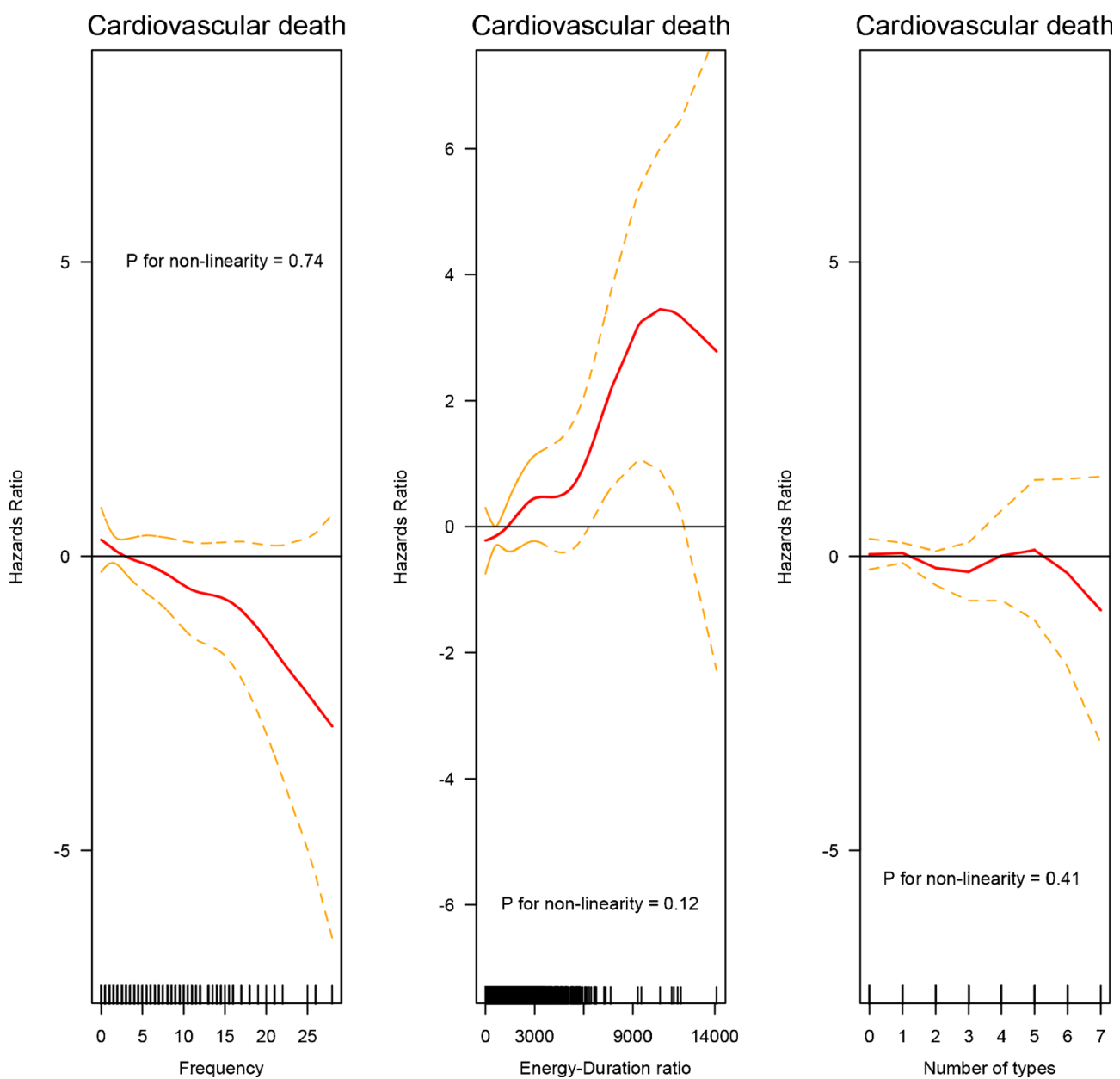

Fig. 2 Assessment of non-linear effects of physical activity on cardiovascular death. The $P$ values indicate a test of non-linearity based on chisquare tests with approximately three degrees of freedom. The dotted yellow lines indicate one standard error from the fitted spline. 
competing risks of cardiovascular and non-cardiovascular death. ${ }^{22}$ This approach is appropriate for addressing epidemiological questions of disease etiology. ${ }^{23}$ We also found evidence that although engagement in a large variety of LTPA is beneficial in reducing mortality, the benefits are non-specific to cardiovascular health.

There are several plausible explanations for why the frequency of activity was protective against cardiovascular mortality. In an older population such as ours, performing frequent LTPA may help maintain or may reflect overall cardiorespiratory fitness, which has strongly protective effects against cardiovascular disease and cancer mortality. ${ }^{24}$ A high frequency of activity is also a marker of an overall healthy lifestyle, which may include diet and regular medical care. Frequent LTPA, like insurance access and education, can also be a proxy for socioeconomic status, in that it requires adequate time and resources. However, the fact that frequency demonstrates an effect on cardiovascular death, but not on non-cardiovascular death, after adjusting for these potential confounders provides insight into the specificity of the impact of frequency on cardiovascular death. These differential effects on cardiovascular versus non-cardiovascular mortality are indicative of the benefit of LTPA frequency on cardiovascular as well as other healthy behaviors. Importantly, a large proportion of our elderly cohort was able to engage in high-frequency activity, indicating the potential public health significance of our findings.

We have demonstrated that a high variety of activity, which itself may contribute to high frequency and total energy, reduced the risk of all-cause mortality. Only a small proportion of our cohort was able to engage in a high variety of activity, so we may have been underpowered to detect a stronger effect on cause-specific mortality. Variety, like frequency, could also act as a proxy for socioeconomic status in terms of having access to a large variety of LTPA resources. However, having the ability to engage in a large number of different activities could be more strongly associated with cardiorespiratory fitness, which may explain why we found a protective effect for all our outcomes.

Our finding that a high EDR was associated with increased cardiovascular mortality merits further discussion. EDR can be viewed as an interaction term between frequency and intensity (average energy per hour). Our findings thus suggest that high frequency of high-intensity exercise may undo the benefits of frequent exercise in terms of cardiovascular mortality. Similar observations were noted by Lee et al. ${ }^{8}$ and Lavie et al.; ${ }^{25}$ they reported that the highest quintile of running characteristics (including intensity and frequency) had a higher risk of cardiovascular mortality than the middle quintiles, whereas our study aimed to parcel out the effects of intensity and frequency. Other potential adverse outcomes of high levels of physical activity, including reduced bone density ${ }^{26}$ and increased risk of cardiac fibrosis, ${ }^{27,28}$ have also been reported. There is also a large body of literature, however, suggesting a cardioprotective effect of high exercise intensity. Tanasescu et al. ${ }^{29}$ showed an inverse relationship between average exercise intensity and risk of coronary heart disease in men. Siscovick et al. ${ }^{30}$ found that exercise intensity was associated with a lower prevalence of subclinical cardiovascular disease markers, especially in women. Manson et al. ${ }^{31}$ reported that high vigorous walking and exercise scores were associated with reduced risk of coronary events in women, although interestingly, the benefits of highly vigorous exercise were similar to those of moderately vigorous exercise.

The current study takes advantage of a data-driven cluster analysis ${ }^{10}$ to derive meaningful LTPA features. Specifically, in addition to frequency and variety, we identified EDR as a subgroup-defining feature. While EDR was shown to have a significant effect on cardiovascular mortality, additional Cox regression analyses found that traditional intensity measure (METS) and total energy were not associated with cardiovascular mortality. In secondary analyses, we accounted for EDR, and the average energy expended was not associated with cardiovascular mortality, while EDR was. This illustrates how cluster analyses can be utilized to extract potentially important features from multidimensional LTPA measurements. This finding may provide additional guidance for refining existing exercise recommendations otherwise not available based on these conventional metrics.

We must add a cautionary note regarding the current study. Because NOMAS represents an elderly population, the range of LTPA may be too limited to provide information of potential harmful effects of extreme levels of exercise. For the same reason, the attenuation effect of high-intensity exercise may not be directly relevant, for instance, to younger individuals who undergo rigorous exercise training. The study also has several limitations. First, we did not directly ask about self-reported strength training, although the questionnaire did include calisthenics. Our primary interest here was not in one specific form of exercise, but rather in the range of activities performed. Second, we did not directly measure cardiorespiratory fitness, which limits the mechanistic understanding of our findings. Similarly, we did not measure the activity performed with actigraphy, though in prior studies both actigraphy and reported LTPA provided important information on health outcomes. ${ }^{32}$ Third, we did not analyze longitudinal LTPA measures. Fourth, while we had $6 \%$ at-home assessment, we did not systematically determine whether the reason was participant frailty, and thus did not have a reliable frailty measure for our analysis. Finally, while three blood pressure readings would be ideal per current guidelines, our measurements were based on two readings per our protocol when the study was initiated in 1993.

In conclusion, our data provide evidence of the central role of frequency and variety of LTPA in reducing cardiovascular mortality in an elderly population. Given the ease of 
participating in low-intensity but daily LTPA, our findings suggest that this can be incorporated into current recommendations provided to older individuals.

Author Contributions: Drs. Cheung and Willey had full access to all the study data and take responsibility for the integrity of the data and the accuracy of the analysis.

Study concept and design: Cheung, Sacco, Elkind, Willey

Acquisition, analysis, or interpretation of data: all authors

Drafting of the manuscript: Cheung, Elkind, Willey

Critical revision of the manuscript for important intellectual content: all authors

Statistical analysis: Cheung, Moon

Study supervision: Sacco, Elkind

Corresponding Author: Joshua Z. Willey, MD, MS; Department of Neurology, Columbia University, 710 West 168th street, New York, NY 10032, USA (e-mail: jzw2@columbia.edu).

\section{Compliance with Ethical Standards:}

Conflict of Interest: The authors declare that they have no conflict of interest.

Funding/Support: This study was supported by the National Institutes of Health grants RO1 HL111195 (Cheung/Elkind), NS K23073104 (Willey), and R01 NSO29993 (Sacco/Elkind).

Role of the Funder/Sponsor: The funding source had no role in the design and conduct of the study with regard to the collection, management, analysis, or interpretation of the data; preparation, review, or approval of the manuscript; or the decision to submit the manuscript for publication.

\section{REFERENCES}

1. Thompson PD, Buchner D, Pina IL, et al. Exercise and physical activity in the prevention and treatment of atherosclerotic cardiovascular disease: a statement from the Council on Clinical Cardiology (Subcommittee on Exercise, Rehabilitation, and Prevention) and the Council on Nutrition, Physical Activity, and Metabolism (Subcommittee on Physical Activity). Circulation. 2003; 107:3109-16.

2. Nelson ME, Rejeski WJ, Blair SN, et al. Physical activity and public health in older adults: recommendation from the American College of Sports Medicine and the American Heart Association. Circulation. 2007; 116: 1094-105.

3. Kiely DK, Wolf PA, Cupples LA, Beiser AS, Kannel WB. Physical activity and stroke risk: the Framingham Study. Am J Epidemiol. 1994; 140:608-20.

4. Lee CD, Folsom AR, Blair SN. Physical activity and stroke risk: a metaanalysis. Stroke. 2003;34:2475-81.

5. Archer E, Blair SN. Physical activity and the prevention of cardiovascular disease: from evolution to epidemiology. Prog Cardiovasc Dis. 2011;53(6):387-96.

6. Sofi F, Capalbo A, Cesari F, Abbate R, Gensini GF. Physical activity during leisure time and primary prevention of coronary heart disease: an updated meta-analysis of cohort studies. Eur J Cardiovasc Prev Rehabil. 2008; 15(3):247-57.

7. Wen CP, Wai JP, Tsai MK, et al. Minimum amount of physical activity for reduced mortality and extended life expectancy: a prospective cohort study. Lancet. 2011;378: 1244-53.

8. Lee DC, Pate RR, Lavie CJ, Sui X, Church TS, Blair SN. Leisure-time running reduces all-cause and cardiovascular mortality risk. J Am Coll Cardiol. 2014;64:472-81.
9. Rosamond W, Flegal $\mathbf{K}$, Furie $\mathbf{K}$, et al. Heart disease and stroke statistics-2008 update: a report from the American Heart Association Statistics Committee and Stroke Statistics Subcommittee. Circulation. 2008;117:e25-146.

10. Cheung YK, Yu G, Wall MM, Sacco RL, Elkind MS, Willey JZ. Patterns of leisure-time physical activity using multivariate finite mixture modeling and cardiovascular risk factors in the Northern Manhattan Study. Ann Epidemiol. 2015;25(7):469-74.

11. McLachlan G, Peel D. Finite Mixture Models. New York: Wiley; 2000.

12. Sacco RL, Anand K, Lee HS, et al. Homocysteine and the risk of ischemic stroke in a triethnic cohort: the Northern Manhattan Study. Stroke. 2004;35:2263-9.

13. Moss AJ, Parsons VL. Current estimates from the National Health Interview Survey: United States, 1985. Vital Health Stat. 10. 1987:i-iv, 1-182.

14. Ainsworth BE, Haskell WL, Whitt MC, et al. Compendium of physical activities: an update of activity codes and MET intensities. Med Sci Sports Exerc. 2000;32:S498-504.

15. Budget. OoMa. Race and ethnic standards for federal statistics and administrative reporting (Directive no 15). 1978:43 Federal Register 19269.

16. Gentry EM, Kalsbeek WD, Hogelin GC, et al. The behavioral risk factor surveys: II. Design, methods, and estimates from combined state data. Am J Prev Med. 1985;1:9-14.

17. Willey JZ, Rodriguez CJ, Moon YP, et al. Coronary death and myocardial infarction among Hispanics in the Northern Manhattan Study: exploring the Hispanic paradox. Ann Epidemiol. 2012;22(5):303-9.

18. Chugh SS, Jui J, Gunson K, et al. Current burden of sudden cardiac death: multiple source surveillance versus retrospective death certificate-based review in a large U.S. community. J Am Coll Cardiol. 2004;44:1268-75.

19. Luna JM, Moon Y, Liu K, et al. Tumour necrosis factor receptor 1 and mortality in a multi-ethnic cohort: the Northern Manhattan Study. Age Ageing. 2013;42(3):385-90.

20. Willey JZ, Rodriguez CJ, Carlino RF, et al. Race-ethnic differences in the association between lipid profile components and risk of myocardial infarction: the Northern Manhattan Study. Am Heart J. 2011;161(5):886-92.

21. Shiroma EJ, Lee I-M. Physical activity and cardiovascular health: lessons learned from epidemiological studies across age, gender, and race/ethnicity. Circulation. 2010;122:743-52.

22. Austin PC, Lee DS, Fine JP. Introduction to the analysis of survival data in the presence of competing risks. Circulation. 2016;133:601-9.

23. Lau B, Cole SR, Gange SJ. Competing risk regression models for epidemiologic data. Am J Epidemiol. 2009;170:244-56.

24. Schmid D, Leitzmann MF. Association between physical activity and mortality among breast cancer and colorectal cancer survivors: a systematic review and meta-analysis. Ann Oncol. 2014;25:1293-311.

25. Lavie CJ, Arena R, Swift DL, et al. Exercise and the cardiovascular system - clinical science and cardiovascular outcomes. Circ Res. 2015;117:207-19.

26. Michel BA, Bloch DA, Fries JF. Weight-bearing exercise, overexercise and lumbar bone density over age 50 years. Arch Intern Med. 1989;149:2325-9.

27. Möhlenkamp S, Lehmann N, Breuckmann F, et al. Running: the risk of coronary events. Prevalence and prognostic relevance of coronary atherosclerosis in marathon runners. Eur Heart J. 2008;29:1903-10.

28. Benito B, Gay-Jordi G, Serrano-Mollar A. Cardiac arrhythmogenic remodeling in a rat model of long-term intensive exercise training. Circulation. 2011;123:13-22.

29. Tanasescu M, Leitzmann MF, Rimm EB, Willett WC, Stampfer MJ, Hu FB. Exercise type and intensity in relation to coronary heart disease in men. JAMA. 2002;288:1994-2000.

30. Siscovick DS, Fried L, Mittelmark M, et al. Exercise intensity and subclinical cardiovascular disease in the elderly. Am J Epidemiol. 1997; 145:977-86.

31. Manson JE, Hu FB, Rich-Edwards JW, et al. A prospective study of walking as compared with vigorous exercise in the prevention of coronary heart disease in women. N Engl J Med. 1999;341:650-8.

32. Colbert LH, Matthews CE, Havighurst TC, Kim K, Schoeller DA. Comparative validity of physical activity measures in older adults. Med Sci Sports Exerc. 2011;43(5):867-76. 